\title{
The effect of ischaemia on the excitability of human sensory nerve
}

\author{
K. N. SENEVIRATNE AND O. A. PEIRIS \\ From the Departments of Physiology and Medicine, Faculty of Medicine, \\ University of Ceylon, Colombo
}

When the limb of a normal subject is compressed by means of a sphygmomanometer cuff, placed on the upper arm and inflated to a pressure beyond the systolic blood pressure, a series of spontaneous sensations are felt in it during and after the release of the vascular occlusion. These sensations felt in the periphery, and collectively referred to as 'pins and needles', have attracted the attention of several investigators, including Lewis, Pickering and Rothschild (1931), Zotterman (1933), Kugelberg (1944, 1946), Weddell and Sinclair (1947), Merrington and Nathan (1949), Poole (1956a, b), and Nathan (1958).

The available evidence suggests that the impulses giving rise to ischaemic and post-ischaemic paraesthesiae arise in the sensory nerves of the limb. There is, however, no consensus of opinion concerning the mechanisms underlying the production of paraesthesiae, and the nature of the sensory nerve fibres involved. Weddell and Sinclair (1947) believed that the post-ischaemic paraesthesiae are due to the stimulation of peripheral nerve endings in the area in which they are felt, and that a greater proportion of the impulses thus aroused are conveyed in the afferent somatic fibres normally subserving the sensation of pain. Merrington and Nathan (1949), however, were of opinion that the nerve impulses felt as paraesthesiae arise in the nerve trunks recovering from the ischaemia, and not in the end organs, and that the fibres concerned are those normally serving the sensations of touch, pressure, and movement.

The evidence suggests that the effect is primarily a vascular one. The ischaemic paraesthesiae are felt during a particular phase of the ischaemic process, while an adequate period of ischaemia must be followed by an adequate period of normal circulation before the nerve becomes the site of the postischaemic paraesthesiae. Kugelberg (1944, 1946) demonstrated an increase in the excitability of human motor nerves at a time when the post- ischaemic paraesthesiae were maximal, and showed that the spontaneous activity in these motor nerves arose when their excitability was raised to a critical value, and that the spontaneous activity ceased when the excitability fell below the critical level. Kugelberg and Cobb (1951) observed that the repetitive tendency and hyperexcitability of the motor nerves were greatly augmented by hyperventilation and hypocalcaemia, and trlieved thato this relationship to changes in the ionic environmen $\overline{\mathbb{C}}$ of the nerve would explain the large range of varia tions in the reaction of normal subjects to ischaemi and post-ischaemic states.

Nathan (1958) suggested that some Group sensory nerve fibres may behave in a similar fashio -that after a few minutes of ischaemia they become sufficiently hyperexcitable for the fibres to fire of $\overrightarrow{\mathscr{C}}$ spontaneously, giving rise to the sensation of $\infty$ ischaemic paraesthesiae. A similar phase of hyperexcitability is believed to occur after the restoration of blood supply to the nerve, this phase lasting longer and being more intense than that occurring during the ischaemic phase.

Poole $(1956 \mathrm{a}, \mathrm{b})$ showed that the occurrence of paraesthesiae, especially those felt during the postischaemic period, is remarkably constant in its time of onset and duration in healthy subjects between the ages of 12 and 60 years. They were, however, absent or diminished in intensity in a group of subjects who had evidence of peripheral sensory nerve disease.

The experiments described below were designed to investigate this hypothesis, by studying the changes of excitability that occurred in the sensory nerve fibres of the distal segment of the median nerve during and after a period of complete vascular occlusion.

\section{METHODS}

Forty healthy males, whose ages ranged from 15 to 45 years, were investigated in this study. 
The sensory nerve potential evoked by stimulation of the digital nerves in the fingers was recorded from the median nerve at the wrist, using a modification of the technique used by Dawson (1956) and Gilliatt and Sears (1958).

Square wave stimuli of variable strength and duration, and at a frequency of $1 / \mathrm{sec}$ from a Grass S4 stimulator were led through a Grass SIU4 RF coupled isolating transformer. The stimulating electrodes were strips of silver $0.8 \mathrm{~cm}$ wide and $5 \mathrm{~cm}$ long, bent to encircle the fingers. Figure 1 illustrates the positions of the stimulating electrodes on the thumb and first three fingers. A metal plate $18 \mathrm{~cm}$ long and $5 \mathrm{~cm}$ wide, bent into a ' $U$ ' shape was used to connect the subject to earth. The hand was placed between the limbs of the metal plate, with one surface in contact with the palmar surface of the hand, and the other in contact with the dorsal surface of the hand.

Two silver discs, each of $1.5 \mathrm{~cm}$ diameter and mounted in a block of Perspex with a distance of $2.5 \mathrm{~cm}$ between their centres, were used for bipolar recording of the median nerve potential. The position of the nerve was located proximal to the wrist skin flexure by palpation of the nerve, or by stimulation and observation of the motor response. The Perspex block with the recording electrodes was then placed over the nerve and held in place by an elastic strap round the wrist.

The surface of the stimulating and recording electrodes, and the earthing plate in contact with skin were coated

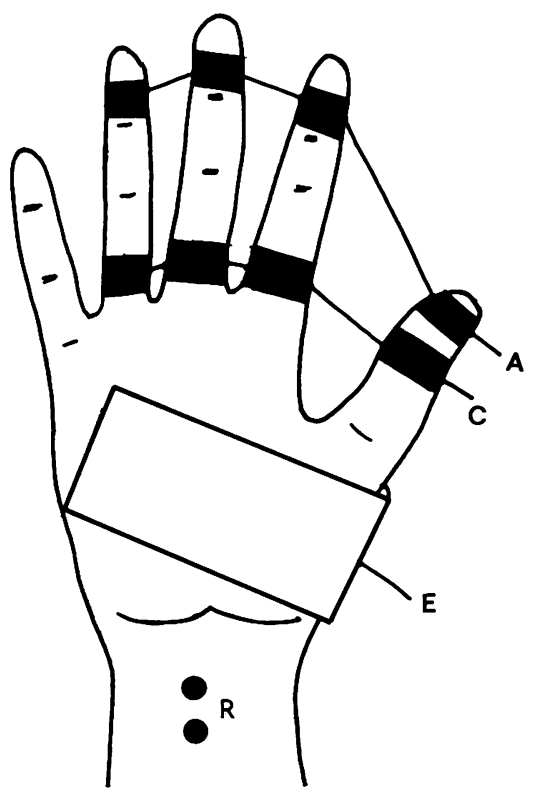

FIG. 1. Positions of the recording and stimulating electrodes on the hand. $A$ and $C$ refer to the anodes and cathodes of the stimulating electrodes, $E$ to the earthing plate, and $R$ to the pair of recording electrodes. with electrode jelly before being placed in position, and great care was taken to prevent accidental smearing of the jelly between the stimulating and recording electrodes, and the earthing plate.

The evoked potentials were amplified by a Grass P511R high-gain RC coupled preamplifier, with its low frequency filter set to produce a half amplitude frequency of $7 \mathrm{c} / \mathrm{s}$ - that is, a time constant of $12 \mathrm{msec}$-and its high frequency filter giving a half amplitude frequency response of $2 \mathrm{kc} / \mathrm{s}$. The amplified responses were monitored through a loudspeaker and displayed on one beam of a Tetronix 502 oscilloscope. The sweep of the oscilloscope was triggered by the stimulator with negligible delay. The lower beam of the oscilloscope carried a $1 \mathrm{msec}$ time signal from a Tetronix 180A signal generator, and also monitored the stimulus through a $10 \mathrm{M} \Omega$ probe. Single sweeps of the oscilloscope were photographed on $35 \mathrm{~mm}$ film with a Cossor camera mounted on the oscilloscope.

The tests were carried out in a room maintained at $27^{\circ} \mathrm{C}$ and all the subjects investigated had an initial inter-digital skin temperature of $31.5^{\circ} \mathrm{C}-32.5^{\circ} \mathrm{C}$ as measured by a skin thermometer. Adequate relaxation was essential to eliminate motor unit activity in the vicinity of the recording electrodes. This was achieved by getting the subject to sit comfortably with his elbow in a semi-flexed position, and with his hand and forearm resting on a soft pillow.

EXPERIMENT 1 Records were taken from all subjects of the response to a supra-maximal stimulus. In this, and all subsequent experiments, the stimulus duration was maintained constant at $0.3 \mathrm{msec}$.

EXPERIMENT 2 The changes in latency and size of response produced by stimuli of increasing voltage were studied in 10 subjects.

EXPERIMENT 3 The reliability of the experimental technique was assessed in five subjects in whom the response to a supra-maximal stimulus was recorded at $1 \mathrm{~min}$ intervals for a period of $1 \mathrm{hr}$.

EXPERIMENT 4 In 10 subjects the response to a supramaximal stimulus was observed on the CRO, and the stimulus strength reduced until the response was approximately $45 \%$ of its maximum size. This stimulus strength was maintained constant and the response to it recorded at $1 \mathrm{~min}$ intervals for a period of $1 \mathrm{hr}$.

Vascular occlusion was obtained by using an ordinary sphygmomanometer cuff placed on the upper arm, with the lower border of the cuff $2 \mathrm{~cm}$ above the medial epicondyle of the elbow. In all cases the resting systolic blood pressure was measured, and occlusion applied by rapidly raising the cuff pressure to $60 \mathrm{~mm}$ above the resting systolic pressure.

EXPERIMENT 5 In five subjects the response to a supramaximal stimulus was recorded. Vascular occlusion was then applied and maintained for $30 \mathrm{~min}$, and records of the response to the stimulus were taken at $1 \mathrm{~min}$ intervals during this period. At the end of $30 \mathrm{~min}$ the cuff was released and the responses recorded at $1 \mathrm{~min}$ intervals for $30 \mathrm{~min}$. 
EXPERIMENT 6 In 15 subjects the stimulus strength was adjusted to give a response approximately $45 \%$ of that obtained with a supra-maximal stimulus. Vascular occlusion was applied and records taken at $1 \mathrm{~min}$ intervals during a $30 \mathrm{~min}$ period of ischaemia, and for a $30 \mathrm{~min}$ period after release of the cuff.

In all experiments in which vascular occlusion was applied, the subjects were told at the beginning of the experiment to report the time of onset, site, and the nature and time of cessation of any subjective sensations they experienced during the ischaemic and postischaemic periods. During the experiment itself they were reminded at regular intervals of the need to report the details of any paraesthesiae they experienced.

\section{RESULTS}

EXPERIMENT 1 The compound sensory nerve potential recorded at the wrist is triphasic (Fig. 2), consisting first of a small positive pre-potential, followed by the much larger negative and positive spikes. Latency measurements were made from the beginning of the stimulus artefact to the peak of the positive prepotential, and response amplitude was measured from the peak of the negative to the peak of the positive spike.

Figure 3 shows the range of potential size in response to a supra-maximal stimulus in $\mathbf{4 0}$ subjects. The potential size varied from $66 \mu \mathrm{V}$ to $170 \mu \mathrm{V}$, with a mean value of $103 \mu \mathrm{V}$, S.D. $\pm 26 \cdot 2 \mu \mathrm{V}$. The mean latency of the response at the wrist was $2.0 \mathrm{msec}$ with a range of $1 \cdot 7-2 \cdot 7 \mathrm{msec}$, S.D. $\pm 0 \cdot 2$ msec.

EXPERIMENT 2 In Experiment 2, the relation between stimulus intensity and response size was determined in 10 subjects, and the results from one subject are reproduced in Figure 4a. These results were used to obtain the data expressed in Fig. 4b, in which size of the response, expressed as a percentage of the maximal response elicited, is plotted as a function of the stimulus intensity.

EXPERIMENT 3 In Experiment 3, five subjects were investigated with respect to the change in size of the response to a supra-maximal stimulus when recorded at $1 \mathrm{~min}$ intervals for $1 \mathrm{hr}$. In none of these did the response vary by more than $\pm 5 \%$ of the mean response value in each subject.

EXPERIMENT 4 In 10 subjects the response to a stimulus evoking a potential approximately $45 \%$ of maximum was recorded for $1 \mathrm{hr}$. These responses did not vary by more than $\pm 10 \%$ of the mean value for each subject. In this, and Experiment 3, stimulus size as monitored on the oscilloscope remained constant throughout each experiment.
EXPERIMENT 5 In five subjects the response to a supra-maximal stimulus was recorded during a 30 min period of complete vascular occlusion and for $30 \mathrm{~min}$ after release of the cuff. The results from one subject are depicted in Figures 5 and 7. It is evident that the response size is little affected during the first $12 \mathrm{~min}$ of ischaemia; it then begins to diminish in size as the ischaemia continues, until, at the 30th minute, it is reduced to $20 \%$ of its

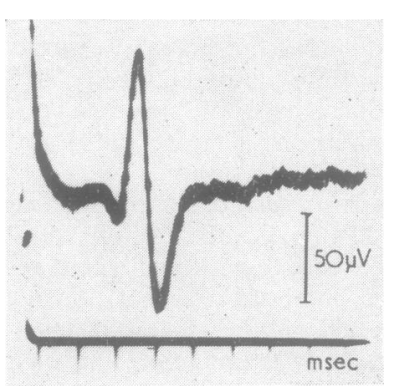

FIG. 2. The triphasic evoked action potential. A small positive pre-potential is followed by the larger negative and positive spikes. The initial upstroke at the commence ment of the time scale indicates stimulus strength.

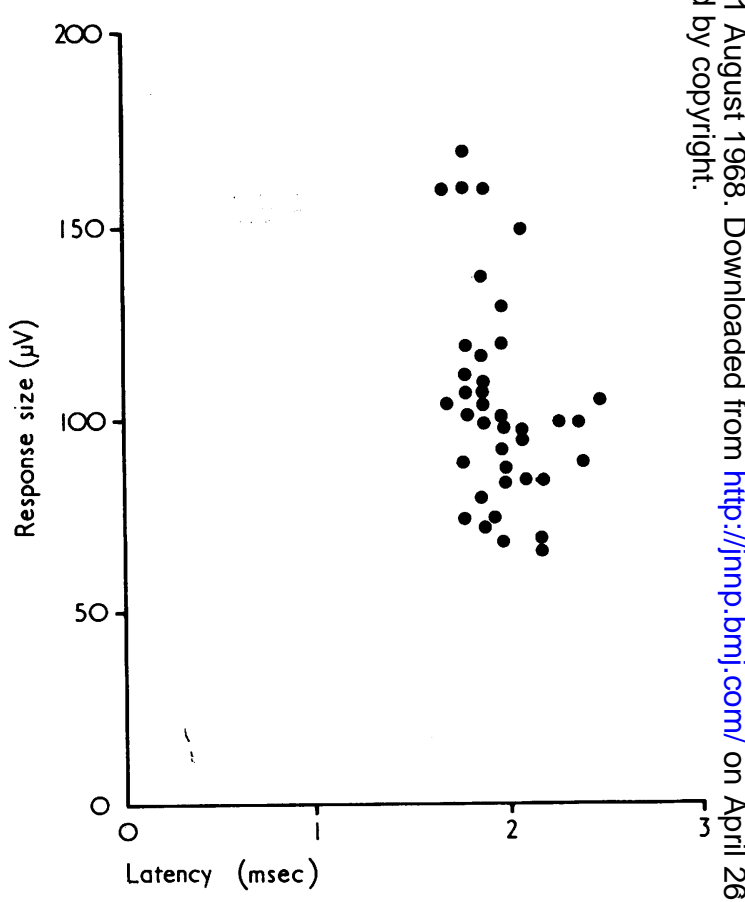

FIG. 3. Latency and size of potential evoked by a supra- N maximal stimulus in $\mathbf{4 0}$ normal subjects. 
initial size. With release of the cuff, the response grows to $76 \%$ of its resting value within the first $2 \mathrm{~min}$, and then continues to grow in size more slowly, until at the end of $30 \mathrm{~min}$ it reaches a size $98 \%$ of normal. The features described here were common to all five experiments of this group. The response remains unaltered for the first 5-12 min of ischaemia, reaches a size $20-30 \%$ of normal at the end of $30 \mathrm{~min}$ of ischaemia, recovers to the extent of $75-85 \%$ within 2 min of release of the cuff, and reaches a value of $95-100 \%$ at the end of $30 \mathrm{~min}$.

EXPERIMENT 6 In this series 15 subjects were investigated; the effect of a stimulus producing a response near $45 \%$ of maximal was recorded during 30 min periods of ischaemia and post-ischaemia.

The five subjects investigated in Experiment 5 were studied again in this group, but the vascular occlusion was applied to the other arm. The results obtained from one such subject appear in Figures 6 and 7. It will be seen that the response grows in size to an extent of $130 \%$ of its resting value within the first few minutes of ischaemia, and then, as the ischaemia continues, becomes progressively smaller, reaching its resting size at the 17 th minute, and becoming negligibly small at the 28th minute. With release of the cuff, the response grows very rapidly in size to $95 \%$ of its resting value by the end of the second minute. During the next $10 \mathrm{~min}$ it remains at a nearly constant size of $106 \%$, then continues to increase in size again, reaching a maximum size of $134 \%$ at the 22 nd minute. At the end of the 30th minute after release of the cuff, the response was still $126 \%$ of its pre-ischaemic size.

Observations on 15 subjects showed that the response increased in size during ischaemia to a mean value of $120 \%$ (range $102-148 \%$ ), and the potential returned to its resting value at the 14th minute (range 8-18 min). At the 30th minute of ischaemia there was no discernible response in 10 of the 15 subjects; in the balance the response was less than $10 \%$ of resting size. At the second minute after release of the cuff, the response had recovered to a mean value of $74 \%$ (range $44-95 \%$ ), and reached a maximum size of $115 \%$ (range $82-154 \%$ ) at the 26th minute (range $15-28 \mathrm{~min}$ ). The results of nine such experiments are shown in Figure 8.

Ischaemic paraesthesiae were reported by all subjects; the mean time of onset was $1.5 \mathrm{~min}$ (range 1.0-2.5 min), and lasted for $6.5 \mathrm{~min}$ (range 5-13 min). Post-ischaemic paraesthesiae of the 'release-pricking' type were also experienced by all subjects; the mean time of onset was $15 \mathrm{sec}$ after release of the cuff (range 12-30 sec) and they lasted for a mean duration of 14.5 min (range 9-20 min).

\section{DISCUSSION}

These results confirm the observations made by Dawson (1956), Gilliatt and Sears (1958), and Buchthal and Rosenfalck (1966) that a small compound nerve action potential can be recorded from the median nerve trunk at the wrist, when brief electrical shocks are applied to the digital
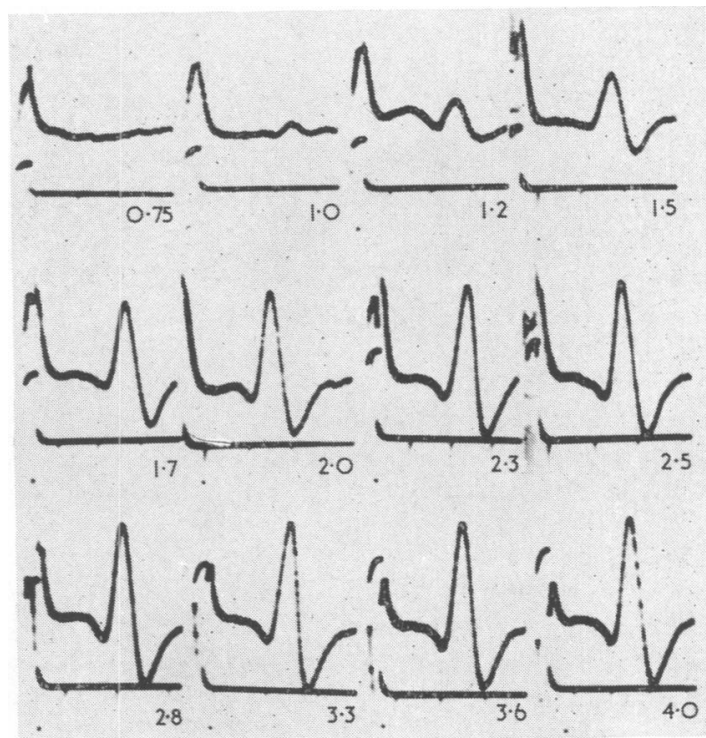

FIG. 4a. Effect of stimulus strength on the evoked response. Figures indicate relative strengths of stimulus.

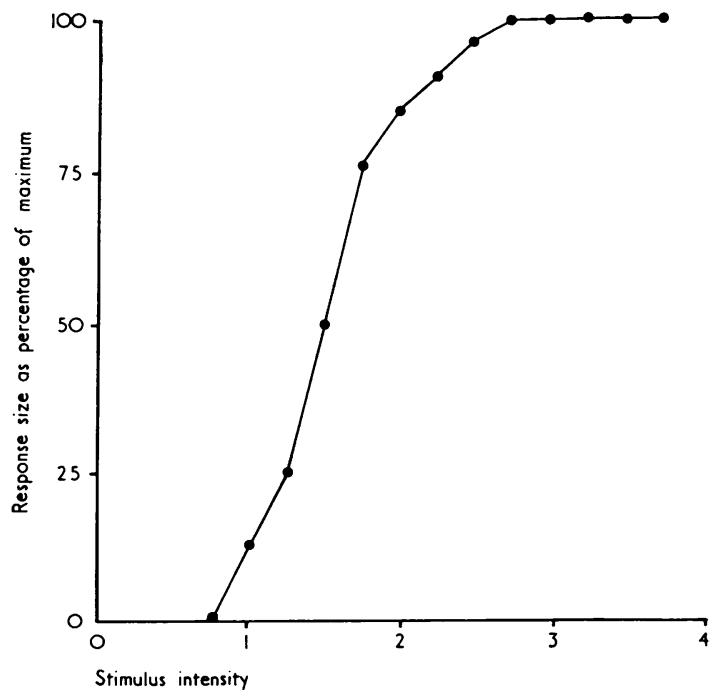

FIG. 4b. Results of Fig. 4 a represented graphically. 
nerves in the fingers. Gilliatt and Sears (1958), recording from surface electrodes at the wrist, stimulated the digital nerves of the index finger in a group of 28 subjects, and recorded a response varying from 9-45 $\mu \mathrm{V}$. Buchthal and Rosenfalck (1966), recording unipolarly from the median nerve at the wrist with a needle electrode, have shown that the recorded potential increases with the number of active nerve fibres, a quantitative relationship being obtained by comparing the response obtained when the fingers were stimulated simultaneously, with that obtained when the fingers $Z_{0}$ were stimulated separately. In 10 observations on subjects between the ages of 16 to 25 years, stimulating the digital nerves in the thumb and first two fingers simultaneously, they obtained responses 0 of $79 \pm 9 \mu \mathrm{V}$ with an S.D. of $30 \mu \mathrm{V}$. Stimulation of the ring finger alone gave a response of $17 \mu \mathrm{V}$. On $\stackrel{\rho}{工}$ simultaneous stimulation of the first three fingers they observed that the response from the nerve at.
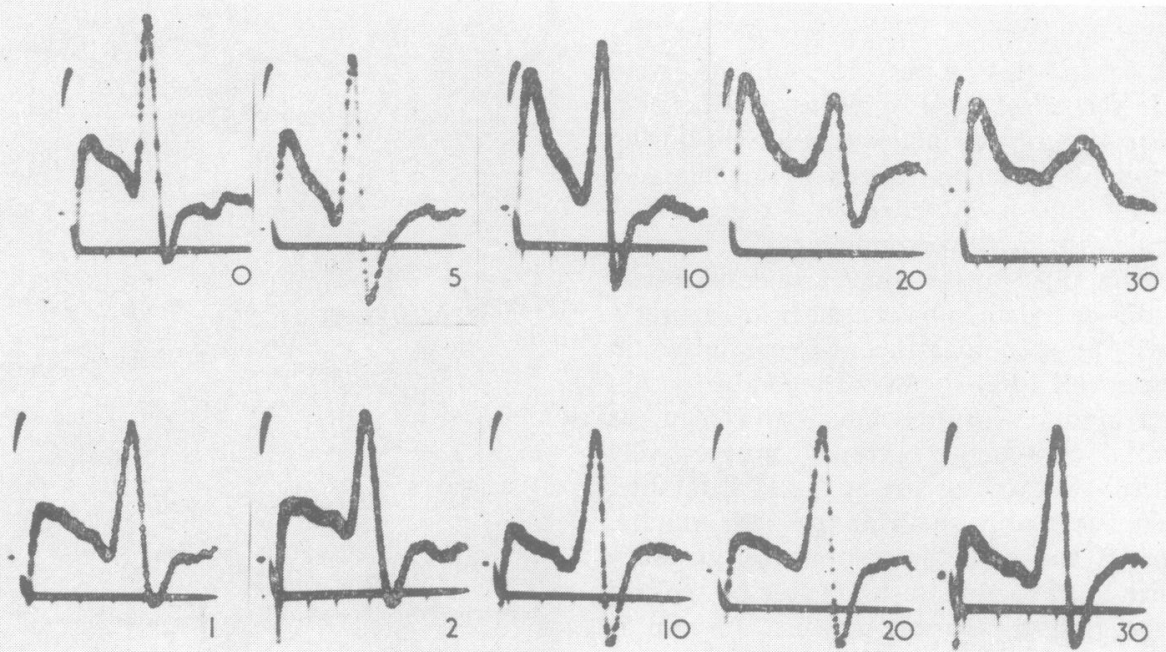

FIG. 5. Effects of ischxemia on the compound action potential evoked by a supra-maximal stimulus. Upper row $=$ during ischaemia, Lower row $=$ after release of cuff. Figures indicate time in minutes.

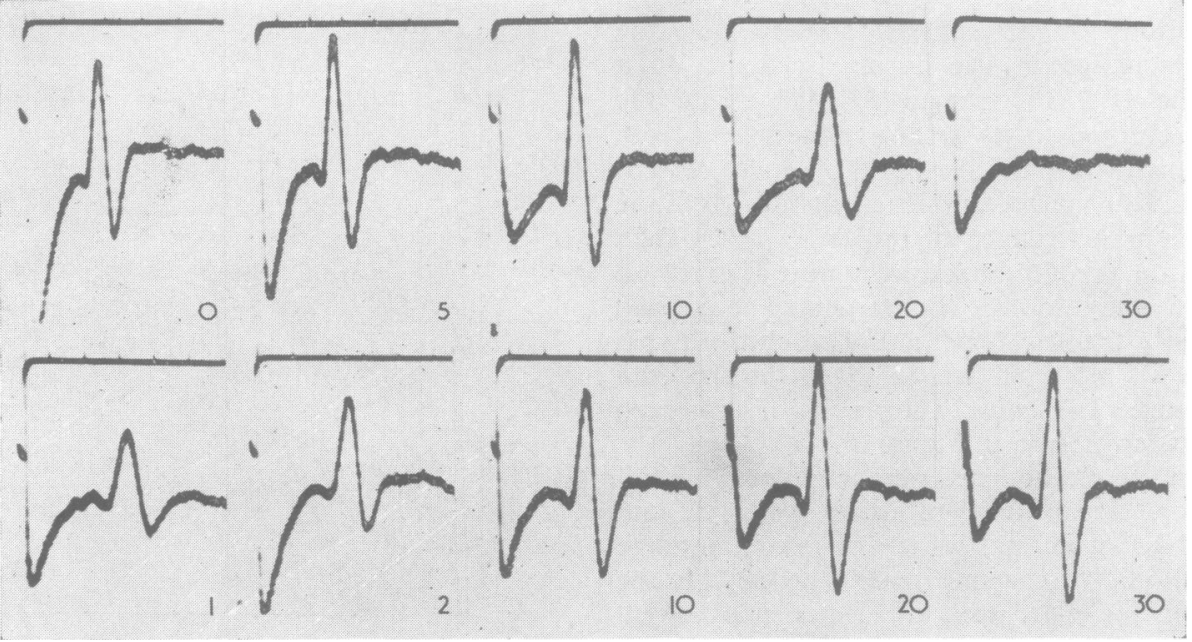

FIG. 6. Effects of ischaemia on the compound action potential evoked by sub-maximal stimulus. Time during and after $N$ ischaemia shown as in Figure 5. 
the wrist was a polyphasic one. The first phase of the response originated from the thumb at a conduction distance of $12 \mathrm{~cm}$, and the second from the index finger at a conduction distance of $17 \mathrm{~cm}$, this polyphasic response being resolved into a triphasic one when the stimulus to the thumb was delayed by $0.6 \mathrm{msec}$.

In our experiments the thumb and three fingers were stimulated simultaneously to obtain a maximally summated potential. A triphasic response was obtained by positioning the stimulating cathodes on the thumb and three figures, so that the conduction distances from stimulating cathodes to the recording electrodes were very nearly equal. The mean maximum response recorded in this way $(103 \mu \mathrm{V} \pm 26 \cdot 2 \mu \mathrm{V})$ corresponds very closely with the maximum summated potential of $96 \mu \mathrm{V}$ obtained by Buchthal and Rosenfalck (1966).

The standard deviation of $25 \%$ of response size in our series corresponds with the $40 \%$ deviation in Buchthal and Rosenfalck's series (Buchthal and Rosenfalck, 1966), and the $30-50 \%$ deviation in Gilliatt and Sears's (1958) series, and is probably due to individual anatomical differences.

Gilliatt, Melville, Velate, and Willison (1965) have investigated the changes in the shape and amplitude of the response when monopolar and

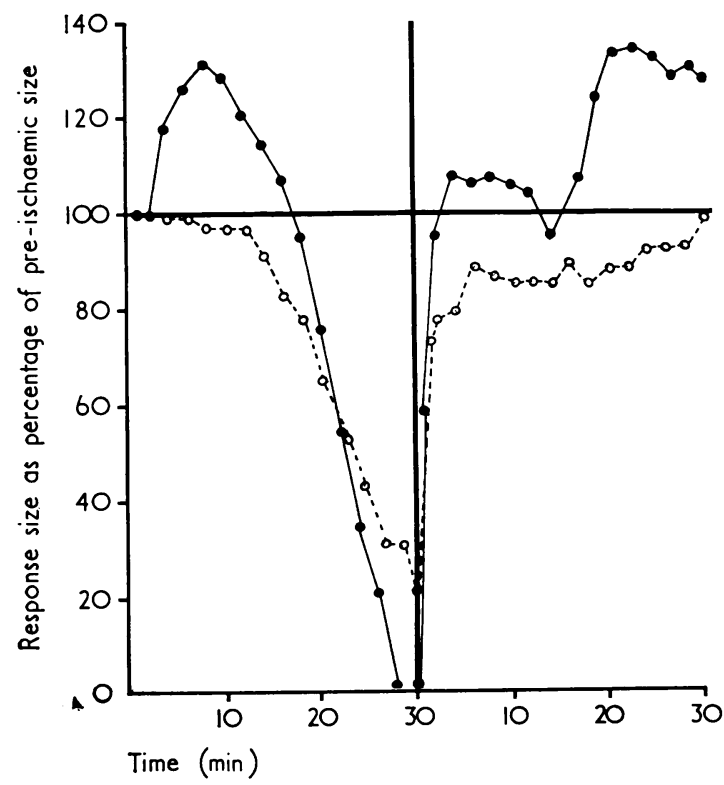

FIG. 7. Change of response size during and after ischaemia as evoked by supra-maximal (open circles) and submaximal (filled circles) stimuli. Figures 5, 6, and 7 show observations made on the same subject. bipolar recording systems were used, and conclude that wave forms recorded from a single active electrode were simpler to interpret than those obtained when the nerve volley passed successively under two active surface electrodes. When a pair of active electrodes was used, their inter-electrode distance affected both the amplitude and duration of the recorded nerve potential, short inter-electrode distances tending to reduce the size of the recorded potential. This arrangement, however, gave a much better rejection of the random activity derived from adjacent muscles. Buchthal and Rosenfalck (1966) have confirmed these findings. Using needle electrodes to record the evoked potential from the median nerve at the wrist, they observed that the response amplitude was maximal when the interelectrode distances were between $30-45 \mathrm{~mm}$, the amplitude being reduced to $50 \%$ of the maximum value when the electrode distance was reduced to $15 \mathrm{~mm}$. In our experiments bipolar surface electrodes with a constant electrode separation of $25 \mathrm{~mm}$ were used; a compromise was effected between the advantage of recording a potential of optimal size with a large inter-electrode distance, and the maximum advantage of common mode rejection of muscle potentials by differential amplification, which necessitates a short electrode separation. The particular features of our experiments favoured the use of a short inter-electrode distance. Since sequential changes were being studied, the conventional technique of photographic superimposition of traces was inapplicable, while the high amplification employed made the rejection of muscle potentials a factor of crucial importance. This was especially necessary, as muscle twitching occurs very commonly during the production of ischaemic and post-ischaemic paraesthesiae.

In our series the mean latency of the response at the wrist was $2.0 \mathrm{msec}$, range $1.7-2.7 \mathrm{msec}$, S.D. $\pm 0.2 \mathrm{msec}$. Gilliatt and Sears (1958) give a range of $2 \cdot 5-4.0 \mathrm{msec}$ in their series of 28 cases; here latency was measured to the peak of the main negative deflection over a conduction distance of $11-15 \mathrm{~cm}$. These latencies, which are significantly longer than those reported in our series, can be accounted for in terms of the delay between the peaks of the positive pre-potential and the main negative spike. Buchthal and Rosenfalck (1966) obtained latencies of 2.5-3.1, $\pm 0 \cdot 1 \mathrm{msec}$, for conduction distances of $12 \cdot 4-17 \cdot 0 \mathrm{~cm}$ in a group of 37 young subjects. In our experiments the conduction distances ranged from $10 \cdot 4-14 \cdot 2 \mathrm{~cm}$.

No attempt was made during this study to determine a distal conduction velocity, because an accurate assessment of conduction distances cannot be made. With supra-maximal stimuli, the assumption 

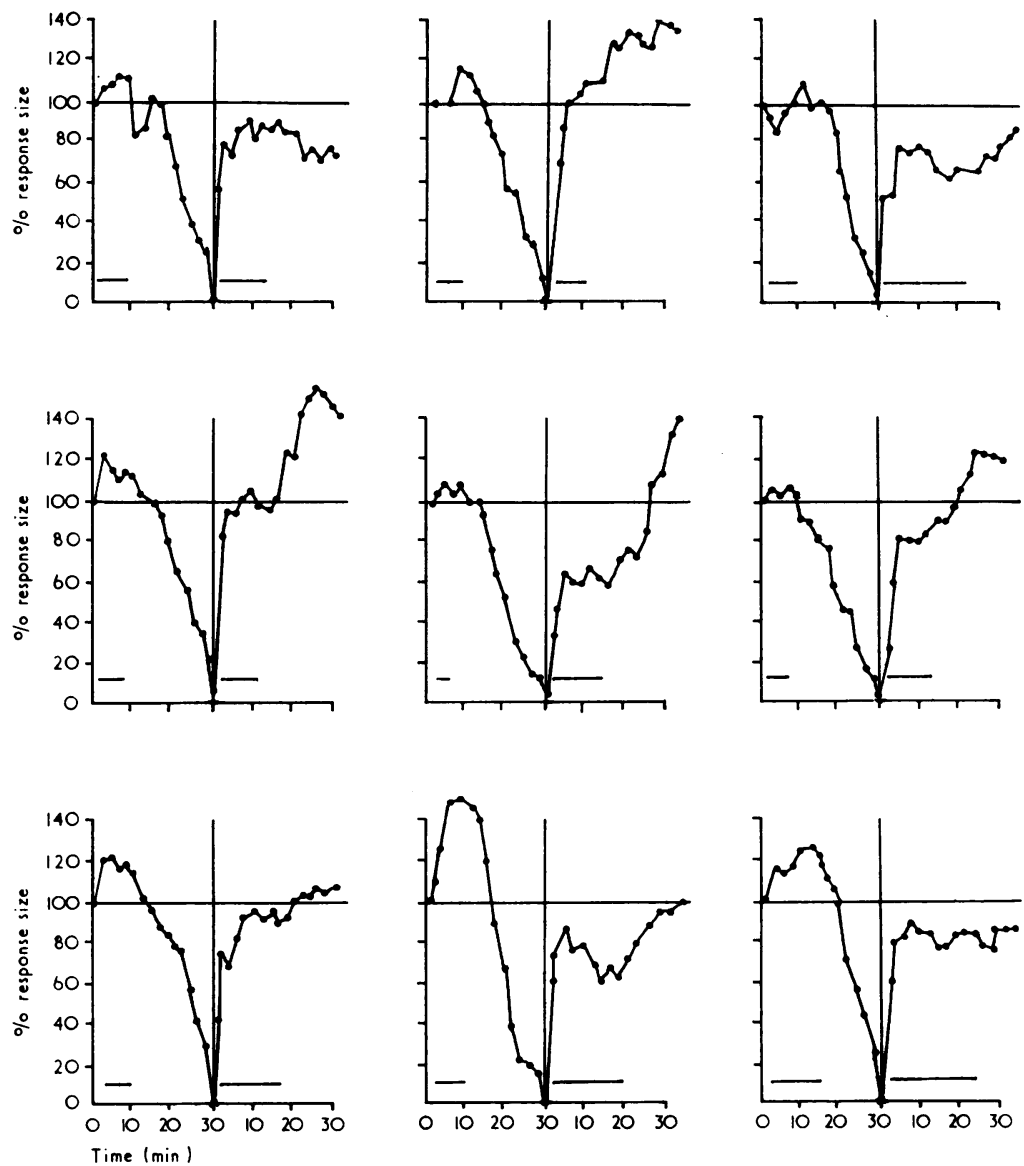

FIG. 8. Results obtained from nine subjects, showing effects of ischaemia on the response to a sub-maximal stimulus. Horizontal lines close to abscissa indicate times of onset and duration of parasthesiae.

that the propagated nerve impulse originates under the stimulating cathode is no longer valid (Rushton, 1949; Gilliat et al., 1965).

It is evident from Figs. $4 a$ and $b$ that, once the stimulus intensity has reached threshold value, further increase of the stimulus strength produces a marked increase in the size of the response, until a critical value of stimulus strength is reached, beyond which the size of the response remains constant. Figure 4b shows that the response grows most markedly in size, from $25-75 \%$ of its maximum value, when the stimulus strength is increased from $0.45-0.63$ of the strength required to elicit a maximal response. This would indicate that there is in the nerve trunk a group of nerve fibres whose thresholds lie within this range, the activity of this group of fibres contributing fully $50 \%$ to the size of the maximum response. Since a relatively large num- ber of fibres share a narrow threshold, changes in $\stackrel{\mathbb{Q}}{\varrho}$ the excitability of this fibre group can best be studied $\overrightarrow{0}$ by using a test stimulus of constant strength which $\exists$ is threshold for approximately half the number of fibres of this group. This feature led to the choice of a test stimulus which gives a response of about $45 \%$ of maximal. With simultaneous stimulation of four fingers, the maximum response is in the region $\frac{0}{0}$ of $100 \mu \mathrm{V}$, and the test stimulus elicits a response of $45 \mu \mathrm{V}$. This potential size is large enough to be $\delta$ recorded at relatively low amplifications, thus $₹$ maintaining a high signal-noise ratio. It follows, 웅 therefore, that an increase in the excitability of fibres ordinarily just beyond the range of this stimulus would reduce their thresholds and render them excitable, while a decrease in the excitability $ᄋ$ of fibres previously excited by the stimulus would $N$ raise their thresholds to a value that now leaves 
them unaffected by the stimulus. Thus an increase in excitability would result in the recruitment of additional fibres, while a decrease would reduce the number of active fibres, and this change would be reflected as a change in the size of the recorded potential.

With the stimulating cathode and anode widely separated on the fingers, it is a reasonable assumption that the larger diameter nerve fibres have a lower threshold than the smaller diameter ones (Rushton, 1951). Histograms of the fibre composition of the digital nerves, examined by electron microscopy (Buchthal and Rosenfalck, 1966), show a bimodal distribution of the fibres with peaks at $3 \mu$ (range 1-3 $\mu$ ) and $9 \mu$ (range 6-13 $\mu$ ). Thomas and Fullerton (1963) gave similar values.

Buchthal and Rosenfalck (1966), however, using a stimulus 30-50 times threshold, could find no evidence of a response derived from the 2-5 $\mu$ fibres. Nor could they, by use of an electronic averaging device, detect evidence of a response derived from the largest diameter fibres. They deduce from a reconstruction of the compound potential that the response recorded at the wrist is made up of the responses of the 6-12 $\mu$ fibres, the initial positive pre-potential being derived from the $11 \mu$ fibres.

Figure 7 illustrates the effects of ischaemia on the excitability of the nerve when the test stimulus used is a maximal one, presumably stimulating all the fibres of the 6-12 $\mu$ group, and when the test stimulus is a sub-maximal one, stimulating only its lower threshold component. It can be seen that supra-maximal stimuli reveal only the decrease of excitability that occurs in the whole nerve, whereas the sub-maximal ones demonstrate the increases and decreases occurring in the low threshold group. Figure 7 shows that an increase in excitabilityas evidenced by an increase in response size-does occur during the early period of ischaemia, at a time when the response to a supra-maximal stimulus is of constant size. After this period the depressant effects of ischaemia become manifest, there is a rapid inactivation of the low threshold fibres, and the response is no longer discernible after the 28 th minute. In 10 out of the 15 subjects studied, the action potential disappeared completely by the 30th minute of ischaemia, while five subjects had an action potential which was less than $10 \%$ of its original size at this time.

These excitability changes occurring in the sensory fibres of the median nerve during ischaemia are very similar to those demonstrated in motor nerve fibres by Kugelberg (1946), while Fullerton (1963) found a demonstrable fall in the motor nerve threshold during the 10th minute of upper limb ischaemia, which was followed by a gradual increase in the threshold during the 20th and 30th minutes of ischaemia.

Figure 7 also shows that a maximal stimulus evokes a response which is reduced to $20 \%$ of its pre-ischaemic size at the end of a $30 \mathrm{~min}$ period of ischaemia, while the response to a sub-maximal stimulus is negligible. This may be due to the low threshold fibres activated by the sub-maximal stimulus being more susceptible to the effects of ischaemia than the high threshold fibres. Alternatively, the persistence of a response to a maximal stimulus may be due to the fact that ischaemia produces an increase in the threshold of all the myelinated fibres of the nerve. A maximal stimulus may therefore continue to excite some of the low threshold fibres which had ceased to respond to a sub-maximal stimulus.

On releasing the cuff, a very rapid rise in the amplitude of the action potential is observed within the first $2 \mathrm{~min}(75-85 \%$ of its original size). Thereafter, the rate of growth of the action potential diminished abruptly in all subjects examined. The beginning of this phase corresponded well with the time of onset of the post-ischaemic paraesthesiae. It seems likely that the first phase of rapid recovery raises the excitability of the fibres of the low threshold group to a critical level, causing them to generate impulses spontaneously. These impulses, when conducted centrally, give rise to the sensation of paraesthesiae, and propagated antidromically down the nerve fibres, render them transiently refractory to the test stimulus applied to the digital nerves. This would reduce the size of the evoked potential recorded at that moment. In these terms, the second phase would correspond to the period of maximum excitability of the nerve fibres. Of the 15 subjects studied, 10 showed a significant increase in the rate of growth of the action potential following cessation of the post-ischaemic paraesthesiae. It is our view that the real increase in size of the action potential following the release of the cuff was masked by the onset of the post-ischaemic paraesthesiae.

These experiments demonstrate that ischaemic and post-ischaemic excitability changes occur in the same group of low threshold fibres, confirming the views of Merrington and Nathan (1949) and Nathan (1958), rather than those of Weddell and Sinclair (1947). The experiments, however, do not define the site of origin of the spontaneously generated impulses. They may arise within the distal segment of the nerve between the recording and stimulating electrodes. Alternatively, as suggested by Merrington and Nathan (1949), they may originate in the segment of the nerve beneath the cuff and travel antidromically into the distal 
segment. Nathan (1958) observed that the sensations of touch and pressure were well preserved in a limb that had been rendered ischaemic for 10-15 min, after which the sensations were progressively obliterated. With restoration of the blood supply there was a very rapid recovery of sensation during the first 1 or 2 min, after which the onset of severe post-ischaemic paraesthesiae masked even the sensation produced by a strong electrical stimulus applied to the digital nerves. This phase of sensory blunting lasted for 3-5 min, normal sensation returning to the limb when the paresthesiae declined in severity. The time course of these events is parallel to the changes of excitability that have been described in our experiments.

Several workers have studied the effects of asphyxia on isolated mammalian nerves (Lehman, 1937; Bentley and Schlapp, 1943; Wright, 1947), while Maruhashi and Wright (1967) have studied the effects of asphyxia on the isolated single rat axon. All these studies indicate that the times taken for impulse transmission to fail, and for restoration of activity on re-oxygenation are in very close agreement with the times taken for ischaemic conduction block and reversal in human limbs, when a pneumatic cuff is used to produce complete vascular occlusion (Magladery, McDougal, and Stoll, 1950; Nathan, 1958). Maruhashi and Wright (1967) find that the spike height of the action potential of the individal axon is well maintained during the first 15-20 min of asphyxia, after which it declines very rapidly in size, and that rapid restitution of potential size takes place on re-oxygenation. The time course of the events is in very close agreement with the results of our experiments. These findings suggest that, since anoxia in vitro and occlusion ischaemia in vivo produce the same effects in the same time, the most important operative factor in occlusion ischaemia must be nerve anoxia.

Wright (1947) has shown that the diminution of the compound nerve potential during asphyxia is due to the reduction in the spike size of individual fibres, failure of transmission in some fibres, and to a disproportionate reduction in conduction velocity producing a rapid temporal dispersion of the summated potential. Our experiments and those of McLeod (1966) confirm the validity of the two latter factors.

\section{SUMMARY}

The digital nerves of the thumb and first three fingers have been stimulated simultaneously and the response evoked by an electrical stimulus recorded from the median nerve at the wrist through surface electrodes.

Stimuli of supra-maximal and sub-maxima strength have been used to investigate the changes in excitability that occur in the nerve during and after a 30 min period of complete vascular occlusion. The results show that during ischaemia a group of low threshold fibres passes through a phase of hyperexcitability before being depressed by the asphyxia. This group of fibres is very susceptible to the effects of asphyxia, their response to this sub $\overrightarrow{\overrightarrow{\vec{f}}}$ maximal stimulus being negligibly small at the end of $30 \mathrm{~min}$ of ischaemia. The same group of fibres recovers its excitability in a characteristic manner verye early in the post-ischaemic period before the onset of post-ischaemic paraesthesiae. Ischaemic and post ischaemic paraesthesiae were experienced by alf who were subject to complete vascular occlusion $\vec{P}$ The times of onset and duration of these sensations related well with observed changes of nerves excitability.

We are greatly indebted to Dr. D. Taverner, reader in Medicine at the University of Leeds, for his very helpfut criticism of this paper.

\section{ADDENDUM}

Since completing this study, our attention has berm

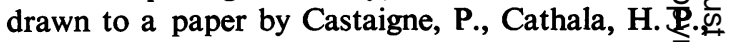
Dry, J. and Mastropaolo, C. (1966) 'Les réspongès des nerfs et des muscles à des stimulations électriqựe: au cours d'une épreuve de garrot ischémique chez l'homme normal et chez le diabétique.'

Rev. neurol., 115, 61-66.

\section{REFERENCES}

Bentley, F. H., and Schlapp, W. (1943). The effects of pressure on conduction in peripheral nerve. J. Physiol. (Lond.), 102, 72-82.

Buchthal, F., and Rosenfalck, A. (1966). Evoked action potentials and conduction velocity in human sensory nerves. Brain Res., 30 1-122.

Dawson, G. D. (1956). The relative excitability and conductio $\bar{D}$ velocity of sensory and motor nerve fibres in Man. $J$. Physiol? (Lond.), 131, 436-451.

Fullerton, P. M. (1963). The effect of ischaemia on nerve conduction in the carpal tunnel syndrome. J. Neurol. Neurosurg. Psychiat.? 26, 385-397.

Gilliatt, R. W., and Sears, T. A. (1958). Sensory nerve action potentialo in patients with peripheral nerve lesions. $J$. Neurol. Neurosurg

Psychiat., 21, 109-118.
-, Melville, I. D., Velate, A. S., and Willison, R. G. (1965). A study of normal action potentials using an averaging technique (barrier grid storage tube). J. Neurol. Neurosurg. Psychiat., 28음 191-200.

Kugelberg, E. (1944). Accommodation in human nerves, and it significance for symptoms in circulatory disturbances ando tetany. Acta physiol. scand., 8, Suppl. 24, 1-105.

(1946). 'Injury activity' and 'trigger zones' in human nerves. O Brain, 69, 310-324. 
- and Cobb, W. (1951). Repetitive discharges in human motor nerve fibres during the post-ischaemic state. J. Neurol. Neurosurg. Psychiat., 14, 88-94.

Lehman, J. E. (1937). The effect of asphyxia on mammalian A nerve fibers. Amer. J. Physiol., 119, 111-120.

Lewis, T., Pickering, G. W., and Rothschild, P. (1931). Centripetal paralysis arising out of arrested blood flow to the limb including notes on a form of tingling. Heart, 16, 1-32.

Magladery, J. W., McDougal, D. B., Stoll, J. (1950). Electrophysiological studies of nerve and reflex activity in normal man. II The effects of peripheral ischemia. Bull. Johns Hopk. Hosp., 80, 291-312.

Maruhashi, J., and Wright, E. B. (1967). Effect of oxygen lack on the single isolated mammalian (rat) nerve fiber. $J$. Neurophysiol. 30, 434-452.

McLeod, J. G. (1966). Digital nerve conduction in the carpal tunnel syndrome after mechanical stimulation of the finger. $J$. Neurol. Neurosurg. Psychiat., 29, 12-22.

Merrington, W. R., and Nathan, P. W. (1949). A study of postischaemic paraesthesiae. J. Neurol. Neurosurg. Psychiat., 12 1-18.

Nathan, P. W. (1958). Ischaemic and post-ischaemic numbness and poraesthesiae. J. Neurol. Neurosurg. Psychiat., 21, 12-23.
Poole, E. W. (1956a). Ischaemic and post-ischaemic paraesthesiae: normal responses in the upper limb with special reference to the effect of age. J. Neurol. Neurosurg. Psychiat., 19, 148-154.

- (1956b). Ischaemic and post-ischaemic paraesthesiae in polyneuritis. J. Neurol. Neurosurg. Psychiat., 19, 281-288.

Rushton, W. A. H. (1949). The site of excitation in the nerve trunk of the frog. J. Physiol. (Lond.), 109, 314-326.

- (1951). A theory of the effects of fibre size in medullated nerve. J. Physiol. (Lond.), 115, 101-122.

Thomas, P. K., and Fullerton, P. M. (1963). Nerve fibre size in the carpal tunnel syndrome. J. Neurol. Neurosurg. Psychiat., 26, 520-527.

Weddell, G., and Sinclair, D. C. (1947). 'Pins and needles': Observations on some of the sensations aroused in a limb by the applicetion of pressure. J. Neurol. Neurosurg. Psychiat., 10, 26-46.

Wright, E. B. (1947). The effects of asphyxiation and narcosis on peripheral nerve polarization and conduction. Amer. J. Physiol., 148, 174-184.

Zotterman, Y. (1933). Studies in the peripheral nervous mechanism of pain. Acta med. scand., 80, 185-242. 